\title{
KIF3C Promotes Proliferation, Migration, and Invasion of Glioma Cells by Activating the PI3K/AKT Pathway and Inducing EMT
}

\author{
Yang Gao, ${ }^{1,2}$ Hui Zheng, ${ }^{3}$ Liangdong Li, ${ }^{1,2}$ Changshuai Zhou, ${ }^{1,2}$ Xin Chen,,2 \\ Xiaoyan Zhou $\mathbb{D}^{2,4}$ and Yiqun Cao ${ }^{1,1,2}$ \\ ${ }^{1}$ Department of Neurosurgery, Fudan University Shanghai Cancer Center, Shanghai 200032, China \\ ${ }^{2}$ Department of Oncology, Shanghai Medical College, Fudan University, Shanghai 200032, China \\ ${ }^{3}$ Department of Nuclear Medicine, Shanghai Tenth People's Hospital, Tongji University, Shanghai 200072, China \\ ${ }^{4}$ Institute of Pathology, Fudan University Shanghai Cancer Center, Shanghai 200032, China
}

Correspondence should be addressed to Xiaoyan Zhou; doctor_md@126.com and Yiqun Cao; gem23@163.com

Yang Gao, Hui Zheng, and Liangdong Li contributed equally to this work.

Received 1 March 2020; Accepted 6 June 2020; Published 23 October 2020

Academic Editor: Wan-Liang Lu

Copyright (c) 2020 Yang Gao et al. This is an open access article distributed under the Creative Commons Attribution License, which permits unrestricted use, distribution, and reproduction in any medium, provided the original work is properly cited.

Kinesin superfamily protein 3C (KIF3C), a motor protein of the kinesin superfamily, is expressed in the central nervous system (CNS). Recently, several studies have suggested that KIF3C may act as a potential therapeutic target in solid tumors. However, the exact function and possible mechanism of the motor protein KIF3C in glioma remain unclear. In this study, a variety of tests including CCK-8, migration, invasion, and flow cytometry assays, and western blot were conducted to explore the role of KIF3C in glioma cell lines (U87 and U251). We found that overexpression of KIF3C in glioma cell lines promoted cell proliferation, migration, and invasion and suppressed apoptosis, while silencing of KIF3C reversed these effects. Ectopic KIF3C also increased the expression of $\mathrm{N}$-cadherin, vimentin, snail, and slug to promote the epithelial-mesenchymal transition (EMT). Mechanistically, overexpression of KIF3C increased the levels of phosphatidylinositol 3-kinase (PI3K) and phosphorylated protein kinase B (p-AKT). These responses were reversed by KIF3C downregulation or AKT inhibition. Our results indicate that KIF3C promotes proliferation, migration, and invasion and inhibits apoptosis in glioma cells, possibly by activating the $\mathrm{PI} 3 \mathrm{~K} / \mathrm{AKT}$ pathway in vitro. KIF3C might act as a potential biomarker or therapeutic target for further basic research or clinical management of glioma.

\section{Introduction}

Glioblastoma multiforme (GBM) is the most common, aggressive, and malignant glioma [1]. Despite comprehensive treatment including maximal surgical resection followed by adjuvant radiotherapy and chemotherapy, GBM patients still suffer from poor prognosis, and the overall median survival is only 14.6 months [2-4]. Thus, it is extremely urgent to explore novel molecular therapeutic targets to improve the overall survival of glioma patients.

Kinesin superfamily proteins (KIFs) are a conserved class of microtubule-dependent motor proteins, which can convert the chemical energy of ATP hydrolysis into mechanical energy to regulate many types of intracellular transport $[5,6]$. KIFs are involved in intracellular organelle/macromolecule transport, cytoskeleton dynamics, and cell division and migration $[7,8]$. The KIF3 family includes KIF3A, KIF3B, and KIF3C $[9,10]$. KIF3A is a microtubule-directed motor subunit that transports the $\beta$-catenin-cadherin complex in the subcellular space [11]. Mouse mutants lacking KIF3A resulted in embryonic lethality as well as large accumulation of arrestin, opsin, and membranes in the retinal photoreceptor inner segment $[12,13]$. In addition, upregulation of KIF3A promotes cell proliferation and invasion in prostate cancer [14]. KIF3B is essential for vesicle transport and membrane expansion during cell mitosis [15]. KIF3B was overexpressed in human hepatocellular carcinoma tissues, and 
TABLE 1: Primary antibodies used in this study.

\begin{tabular}{|c|c|c|c|c|}
\hline Primary source & Antibody clone & Company & Code & Dilution \\
\hline KIF3C & Rabbit & Proteintech & 14333-1-AP & $1: 1000$ \\
\hline PI3K & Rabbit & CST & 4257 & $1: 1000$ \\
\hline $\mathrm{AKT}$ & Rabbit & CST & 4691 & $1: 1000$ \\
\hline p-AKT & Rabbit & CST & 4060 & $1: 1000$ \\
\hline Vimentin & Rabbit & CST & $5741 \mathrm{P}$ & $1: 1000$ \\
\hline Slug & Rabbit & CST & $4585 \mathrm{P}$ & $1: 1000$ \\
\hline Snail & Rabbit & CST & $38879 \mathrm{P}$ & $1: 1000$ \\
\hline BCL-2A1 & Rabbit & CST & $35596 / 4926$ & $1: 1000$ \\
\hline BAX & Rabbit & Proteintech & 50599-2-lg & $1: 1000$ \\
\hline Cleaved caspase-3 & Rabbit & CST & $9664 \mathrm{~T}$ & $1: 1000$ \\
\hline Caspase-3 & Rabbit & Abcam & ab13585 & $1: 1000$ \\
\hline GAPDH & Rabbit & Proteintech & 10494 & $1: 10000$ \\
\hline $\mathrm{N}$-cadherin & Rabbit & Proteintech & 22018-1-AP & $1: 1000$ \\
\hline
\end{tabular}

Abbreviation: KIFs: kinesin superfamily proteins; PI3K: phosphatidylinositol 3-kinase; AKT: protein kinase; GAPDH: glyceraldehyde-3-phosphate dehydrogenase; CST: Cell Signaling Technology.

TABLE 2: Secondary antibodies used in this study.

\begin{tabular}{lcc}
\hline Secondary antibody & Dilution & Company \\
\hline Anti-mouse & $1: 3000-1: 5000$ & Proteintech \\
Anti-rabbit & $1: 3000-1: 5000$ & Proteintech \\
\hline
\end{tabular}

its downregulation might inhibit hepatocellular carcinoma proliferation [16]. Another research has shown that KIF3A is related to KIF3B or KIF3C, while KIF3B and KIF3C have no interaction with each other [17].

In contrast to KIF3A and KIF3B, KIF3C is expressed in the central nervous system (CNS). Its expression is identified not only in neuronal cells but also in astrocytes. These findings suggest that the multiple transport roles of KIFs are common in neurons and glial cells $[18,19]$. Until now, little information is available on KIF3C in tumor diseases and CNS disorders. The previous report indicated that KIF3C was an injuryspecific kinesin that organized the microtubule cytoskeleton in the growth cone to regulate axon growth and regeneration after injury [20]. Wang et al. found that KIF3C was overexpressed in breast cancer tissues, and downregulation of KIF3C could suppress tumor growth and metastasis in breast cancer by inhibiting TGF- $\beta$ signaling [21]. The upregulation of KIF3C has been identified during neural differentiation [22]. However, the exact function and possible mechanism of the motor protein $\mathrm{KIF} 3 \mathrm{C}$ in glioma remain unclear.

In this study, we conducted an in vitro study to investigate the function and mechanism of KIF3C in the process of proliferation, migration, invasion, and apoptosis in glioma cells.

\section{Materials and Methods}

2.1. Cell Culture. The U87 and U251 cell lines were purchased from the cell library of the Chinese Academy of Sciences. All cell lines were cultured in Dulbecco's modified Eagle's medium (DMEM) (Gibco BRL, Gaithersburg, MD, USA) supplemented with $10 \%$ fetal bovine serum (Gibco), gluta- mine ( $2 \mathrm{mM}$, Gibco), streptomycin $(100 \mu \mathrm{g} / \mathrm{ml}$, Gibco), and penicillin (100 U/ml, Gibco). The cultures were incubated at $37^{\circ} \mathrm{C}$ in a humidified $5 \% \mathrm{CO}_{2}$ atmosphere.

2.2. Western Blot Analysis. Cellular proteins were extracted by using radioimmunoprecipitation assay (RIPA) buffer containing a protease inhibitor cocktail (BestBio, Shanghai, China). Equal amounts of protein were subjected to sodium dodecyl sulfate polyacrylamide gel electrophoresis (SDSPAGE) and transferred to polyvinylidene fluoride (PVDF) membranes (Millipore, Billerica, MA, USA). The membranes were blocked with blocking buffer (5\% nonfat milk powder in TBS containing $0.1 \%$ Tween) for $2 \mathrm{~h}$. Then, the membranes were incubated with various primary antibodies followed by the appropriate horseradish peroxidase- (HRP-) conjugated secondary antibodies. Additionally, enhanced chemiluminescence (ECL) (Millipore, Billerica, MA, USA) was used to identify immunoreactive bands. All experiments were repeated three times. The antibodies used in this study are listed in Tables 1 and 2.

2.3. Quantitative Real-Time PCR. Total RNA was extracted with the TRIzol reagent (Thermo Fisher, Shanghai, China) and reverse transcribed using All-in-One qPCR Mix (AT341, China). Subsequently, real-time PCR was performed with SYBR Premix Ex Taq (TaKaRa Biotechnology, Dalian, China) using the Illumina Eco Real-Time PCR System (Thermo Fisher, Shanghai, China). The primer sequences used in this study are as follows:

(i) GAPDH: F 5' -GGAGCGAGATCCCTCCAAAAT-3'

(ii) GAPDH: $\mathrm{R}$ 5'-GGCTGTTGTCATACTTCTCAT GG-3'

(iii) KIF3C: F $5^{\prime}$-CCGGGCCTCCTATTTGGAGA-3'

(iv) KIF3C: R 5' -TCCTTGATGTAGACGCCAGTC-3' 

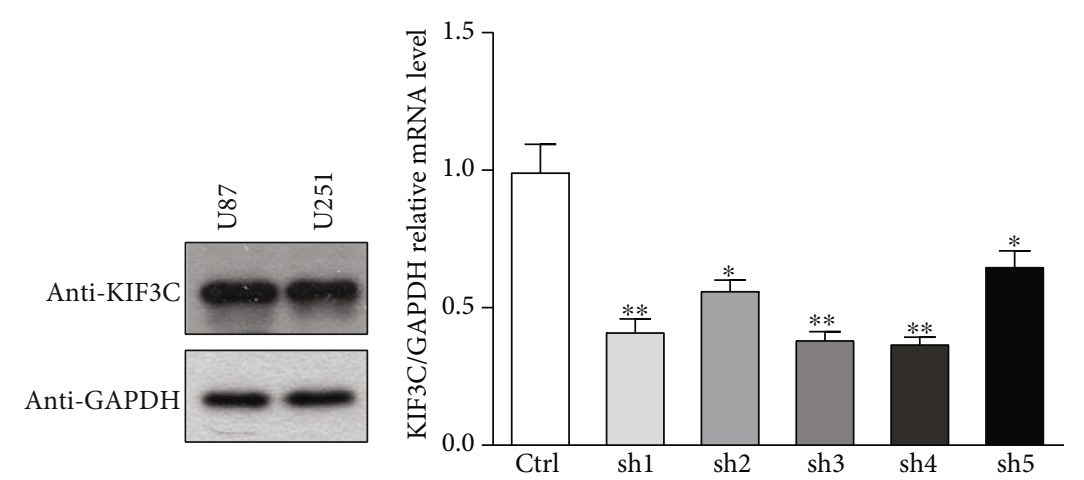

(a)

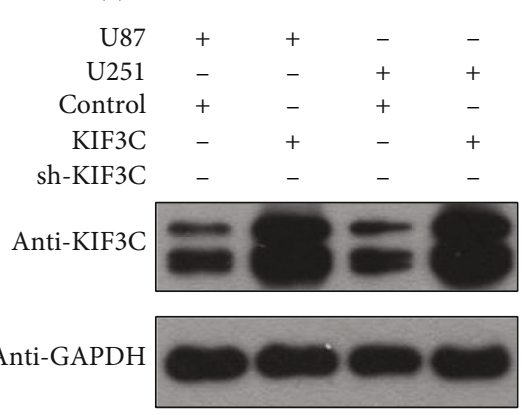

(c)

(b)

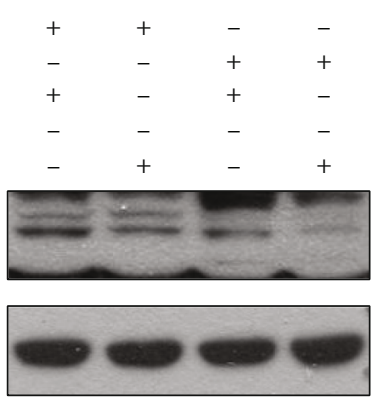

FIGURE 1: Expression of KIF3C in glioma cell lines. (a) Baseline expression of KIF3C in U87 and U251 cells. (b) Silencing efficiency evaluated by real-time PCR analysis. (c) Silencing and overexpression efficiency of KIF3C evaluated by western blotting analysis in glioma cells. GAPDH was used as the loading control.

2.4. Transient Transfection. Human KIF3C cDNA was subcloned from the glioma cell lines U87 and U251 into the lentiviral vector pCDH-CMV-MCS-EF1-Puro (GeneChem, Shanghai, China). The cloned primer sequences are as follows:

\section{(i) F: 5'-ATGGCCAGTAAGACCAAGGC-3'}

(ii) R: 5' -TCAAGCGTAGTCTGGGACGT-3'

KIF3C-shRNAs were purchased from GeneChem and expressed in the vector pGIPZ (GeneChem, Shanghai, China). The target sequences are as follows:

(i) shRNA-1: 5' -AGGAGATTGCCGAGCAGAA-3'

(ii) shRNA-2: 5' $^{\text {-TCGCTAAACGAAGATATTA-3' }}$

The empty vector was used as the negative control.

2.5. Migration Assay. A cell migration assay was examined by using a 24-well unit containing $8 \mu \mathrm{m}$ (pore size) polycarbonate membrane inserts (BD Biosciences, San Jose, CA). Approximately $2.0 \times 10^{4}$ cells were added to the upper chamber in medium, and $600 \mu \mathrm{l}$ of culture media containing $20 \%$ fetal bovine serum was added to the bottom chamber. Cells were allowed to migrate at $37^{\circ} \mathrm{C}$ for $24-48 \mathrm{~h}$ toward the lower reservoir. Cells in the upper chambers were removed, and cells in the bottom chambers were fixed with paraformalde- hyde and stained with crystal violet. The experiments were repeated three times with duplicate samples.

2.6. Invasion Assay. A transwell chamber invasion assay (Matrigel-coated membrane, BD Biosciences, San Jose, CA) was used to examine the cell invasion assay. Approximately $2.0 \times 10^{4}$ cells were seeded in serum-free medium into the upper chamber and allowed to invade the lower chamber, which contained culture medium with $20 \%$ fetal bovine serum. After $24-48 \mathrm{~h}$, cells invading the matrix were then fixed with paraformaldehyde for 15 minutes and stained with crystal violet for 30 minutes. All experiments were repeated three times.

2.7. Cell Proliferation Assay. Cell Counting Kit-8 (CCK-8, Dojindo Molecular Technologies) was used for test cell proliferation assays. U87 (control and transfection of KIF3CsiRNA and KIF3C overexpression vectors) and U251 (control and transfection of KIF3C-siRNA and KIF3C overexpression vectors) cells were seeded in 96-well plates at a density of $2 \times 10^{4}$ cells/well in $100 \mu \mathrm{l}$ of medium and grown overnight. At the indicated time points, CCK- 8 was used to measure cell proliferation indices according to the manufacturer's instructions. Next, a 96-well format plate reader (Tecan Sunrise, Switzerland) was adopted to measure the cell numbers in triplicate by measuring the absorbance at a wavelength of $450 \mathrm{~nm}$ (OD450) every $12 \mathrm{~h}$ for 3 days. The experiments were repeated three times. 


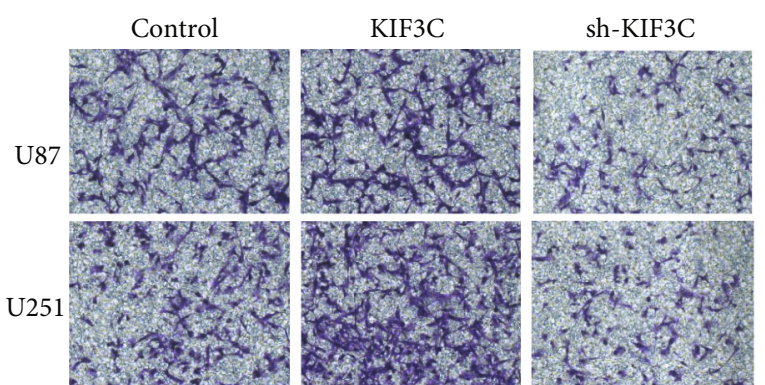

(a)

KIF3C

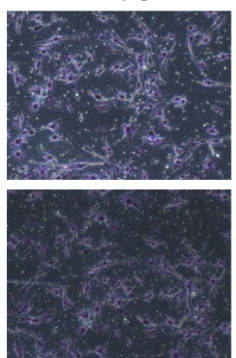

(c)

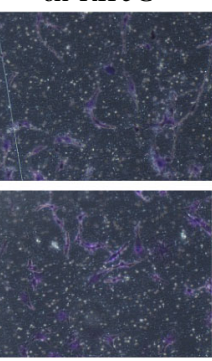

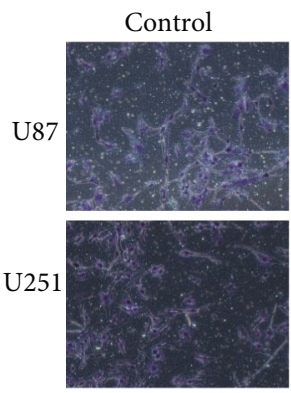

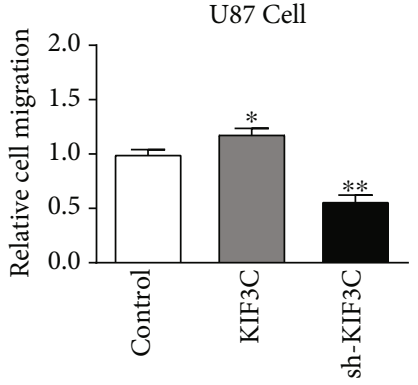

(b)
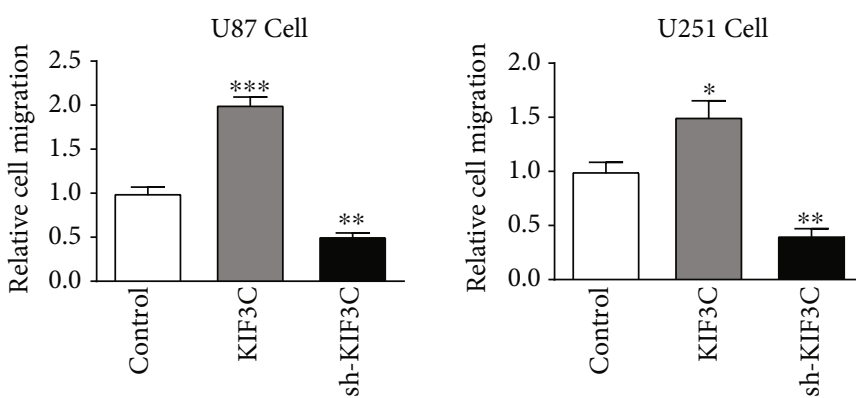

(d)

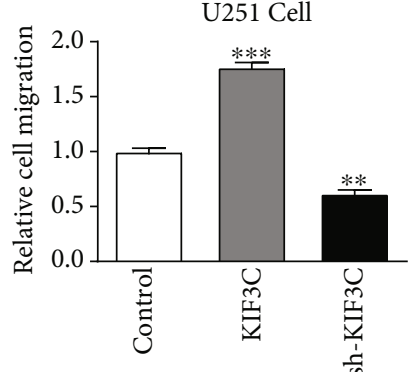

U251 Cell

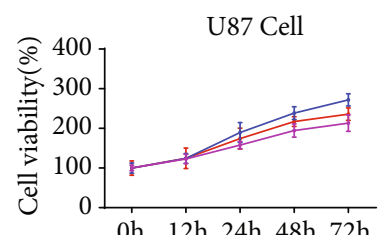

0h $12 \mathrm{~h} 24 \mathrm{~h} 48 \mathrm{~h} 72 \mathrm{~h}$

$\rightarrow$ Contol

$\rightarrow \mathrm{KIF} 3 \mathrm{C}$

$\rightarrow$ shKIF3C
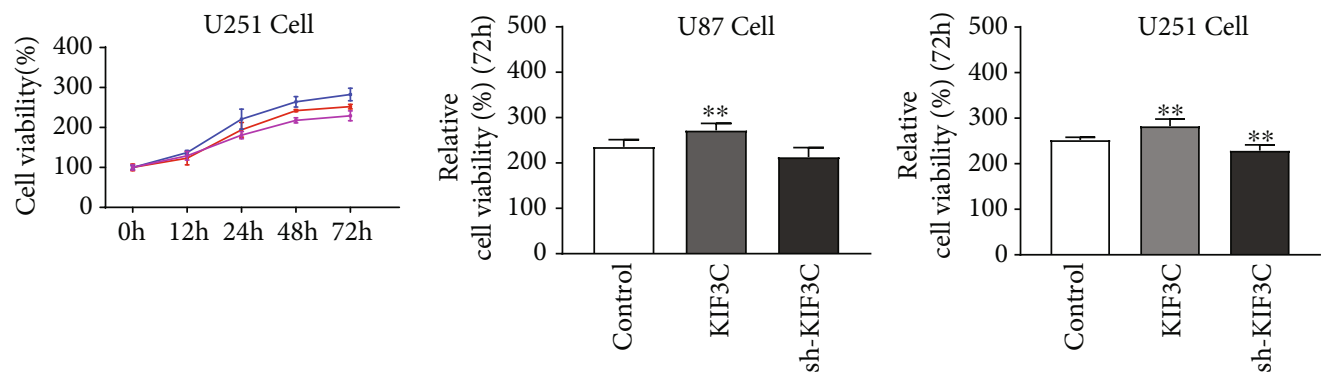

(e)
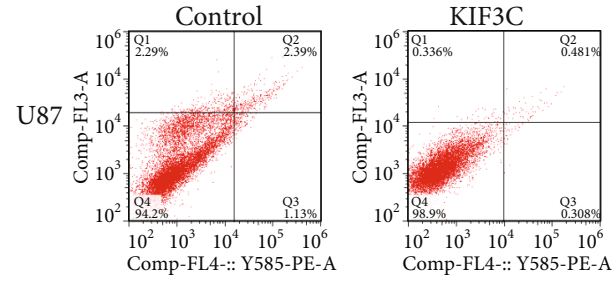

(f)
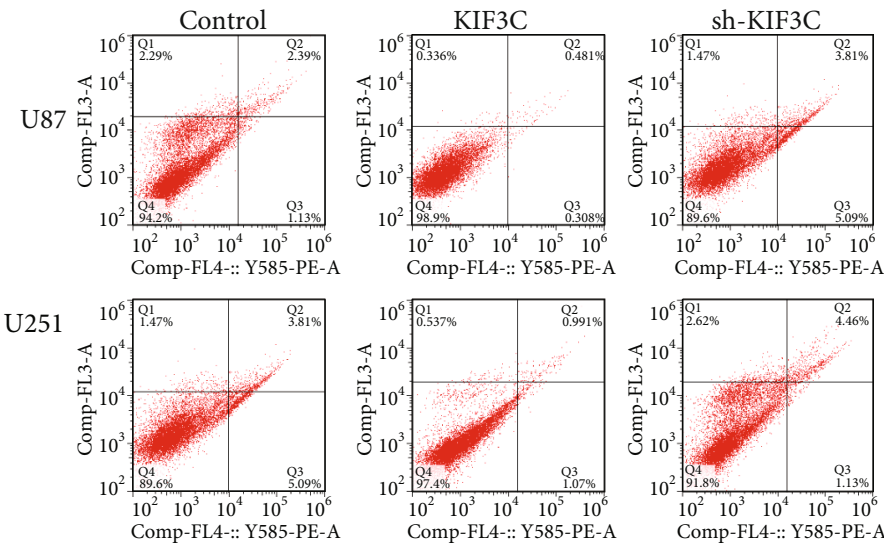

(g)

Figure 2: Continued. 

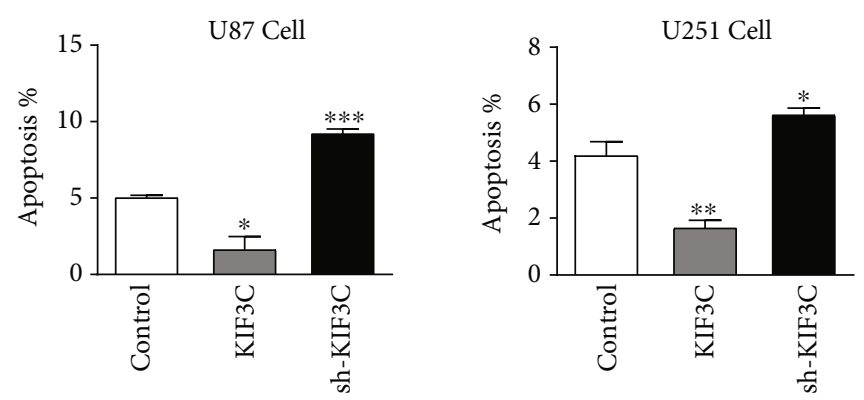

(h)

Figure 2: KIF3C promotes U87 and U251 cell migration, invasion, and proliferation and suppresses cell apoptosis in vitro. (a, b) Migration and $(c, d)$ invasion of each cell line were evaluated by the transwell assay. The left panels show photos of representative fields (100x magnification), and the right panels show histograms of the results. (e, f) Proliferation was determined at the indicated time intervals using the Cell Counting Kit-8 reagent. (g, h) Cell apoptosis was measured using the annexin V-FITC/PI apoptosis detection kit. Data are expressed as the mean \pm SD. Each experiment was repeated three times. ${ }^{*} P<0.05$, ${ }^{* *} P<0.01$, and ${ }^{* * *} P<0.001$.

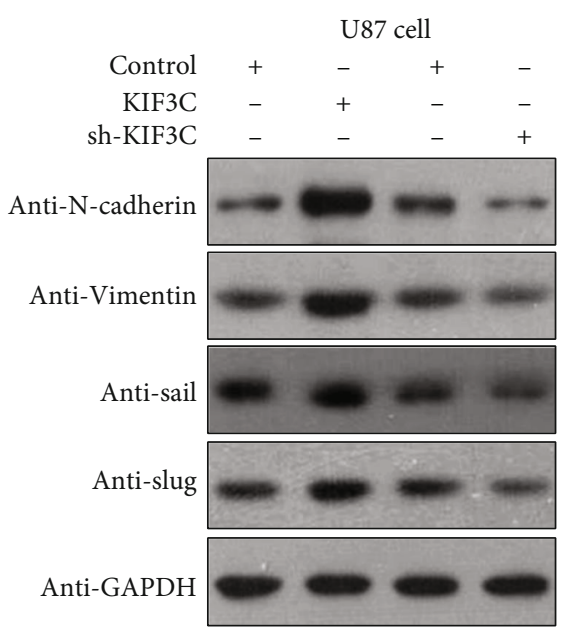

(a)

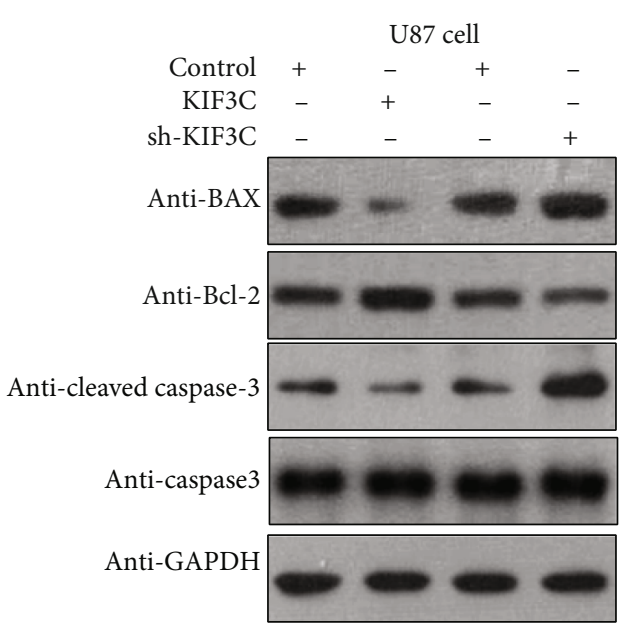

(c)

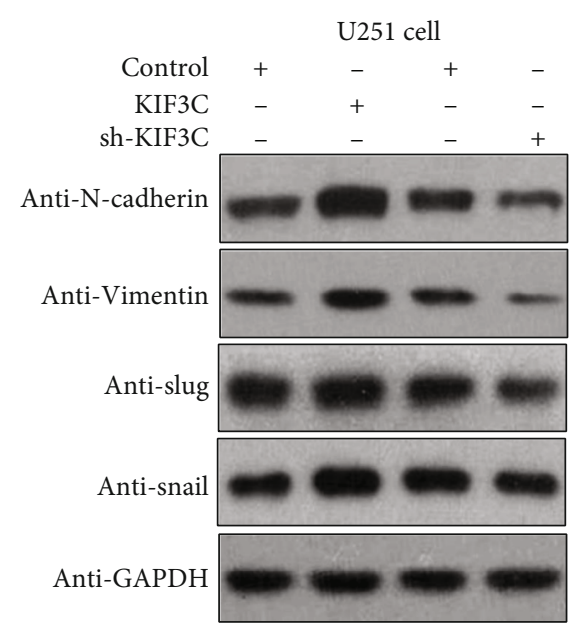

(b)

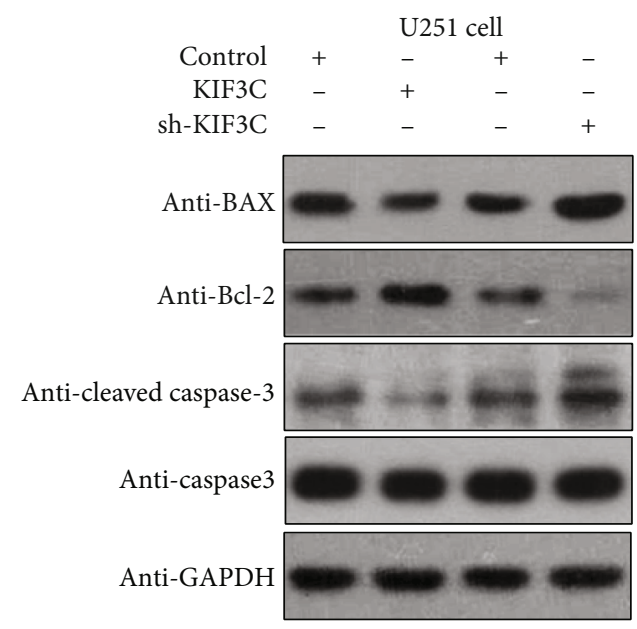

(d)

FIGURE 3: KIF3C modulates the expression of EMT and apoptosis-related proteins in vitro. (a) Overexpression of KIF3C increased Ncadherin, vimentin, snail, and slug expression to promote EMT in vitro, while KIF3C downregulation had the opposite effects. (b) Loss of KIF3C increased Bax levels and decreased Bcl-2 and cleaved caspase- 3 expression, while overexpression of KIF3C had the opposite effects. (c, d) The same results were also found in U251 cells. 


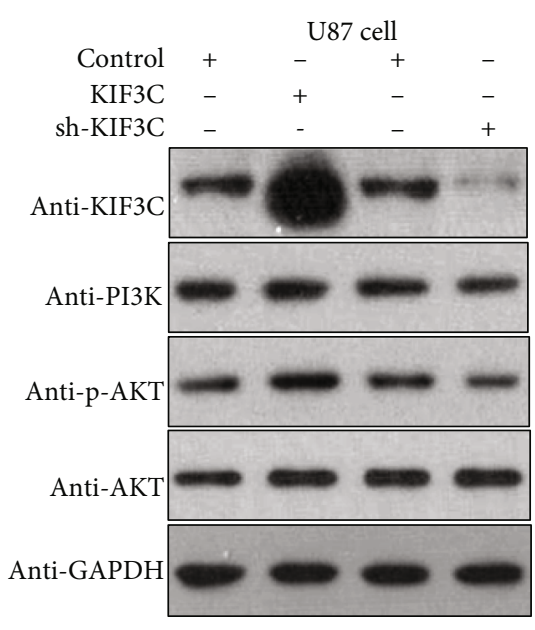

(a)

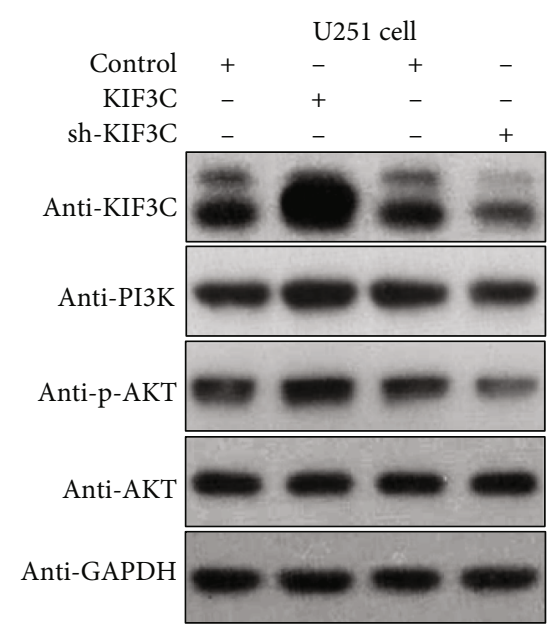

(b)

FIGURE 4: KIF3C was related to the PI3K/AKT pathway. (a, b) Western blotting demonstrated that overexpression of KIF3C increased PI3K and p-AKT level, while KIF3C downregulation had the opposite effects.

2.8. Apoptosis Assay. Cell apoptosis was measured by using the annexin V-FITC/PI apoptosis detection kit (BD Biosciences) according to the manufacturer's protocol. The mixture was then shaken thoroughly in a dark room at room temperature for 15 minutes and supplemented with $10 \mu \mathrm{l}$ PI. Flow cytometry (Beckman Coulter, Inc.) was utilized to analyze cell apoptosis. All experiments were repeated three times.

2.9. Statistical Analysis. Statistical analyses were performed with GraphPad Prism 6 and SPSS software 17.0 (SPSS Inc., Chicago, IL, USA). All data are shown as the mean \pm SD. In addition, Student's $t$-test or one-way ANOVA was used to evaluate differences between groups. $P$ values $<0.05$ were considered statistically significant.

\section{Results}

3.1. Expression of KIF3C in Glioma Cell Lines after RNA Interference or Plasmid Transfection. The level of KIF3C expression was identified by western blot analysis in U87 and U251 glioma cell lines (Figure 1(a)). To investigate the role of KIF3C in vitro, we used an shRNA targeting KIF3C in U87 and U251 cell lines (Figure 1(b)). The shRNA construct strongly reduced the level of KIF3C protein in both cell lines (Figure 1(c)). In addition, the KIF3C ctDNA pCDHCMV-MCS-EF1-Puro vector was transfected into U87 and U251 cell lines for the gain-of-function study. The empty vector was used as a negative control. Western blot analysis showed that KIF3C was increased in the transfection group compared to the empty vector group (Figure 1(c)).

\subsection{Effects of KIF3C on the Migration and Invasion of Glioma} Cells. As shown in Figures 2(a) and 2(b), cell migration in the KIF3C overexpression groups was significantly higher than that in the negative control groups, while the shRNAKIF3C groups had the opposite effect on the two cell lines. Glioma cells had a higher invasive ability after increasing
KIF3C expression, while the shRNA-KIF3C group had a less invasive ability in the two cell lines (Figures 2(c) and 2(d)). These results suggest that KIF3C can promote the invasive and migratory ability of U87 and U251 cells.

3.3. Effects of KIF3C on Proliferation and Apoptosis of Glioma Cells. The results showed that overexpression of KIF3C promoted U87 cell proliferation, while downregulation of KIF3C inhibited this effect compared to that of the control group (Figures 2(e) and 2(f)). Moreover, elevated KIF3C expression reduced U87 cell apoptosis, while suppressed KIF3C expression promoted U87 cell apoptosis (Figures 2(g) and 2(h)). KIF3C had the same effects on U251 cell lines (Figures 2(g) and 2(h)). These results suggest a potential role for KIF3C in promoting cell proliferation and suppressing cell apoptosis.

3.4. KIF3C Modulates the Expression of EMT and ApoptosisRelated Proteins. We found that in the GBM cell lines, the KIF3C upregulation group had increased expression levels of N-cadherin, vimentin, snail, and slug, inducing EMT, while KIF3C downregulation had the opposite effects (Figures 3(a) and 3(b)). Tumor cell growth relies on antiapoptotic mechanisms or activation of survival signals, such as the PI3K/AKT pathway. Bax has been demonstrated as an apoptosis promoter, while $\mathrm{Bcl}-2$ and cleaved caspase- 3 are apoptosis inhibitors. In our study, the KIF3Cdownregulating cell group exhibited elevated levels of Bax and decreased levels of Bcl-2 and cleaved caspase- 3 expression, while overexpression of KIF3C had the opposite effects on the two cell lines (Figures 3(c) and 3(d)).

3.5. KIF3C Regulates the PI3K/AKT Pathway in Glioma Cell Lines. We further explored the mechanisms of KIF3C on proliferation, migration, and invasion in glioma cells. The reports indicated that the $\mathrm{PI} 3 \mathrm{~K} / \mathrm{AKT}$ signaling pathway is activated to promote cell proliferation, migration, and invasion in GBM $[23,24]$. Therefore, we investigated whether 

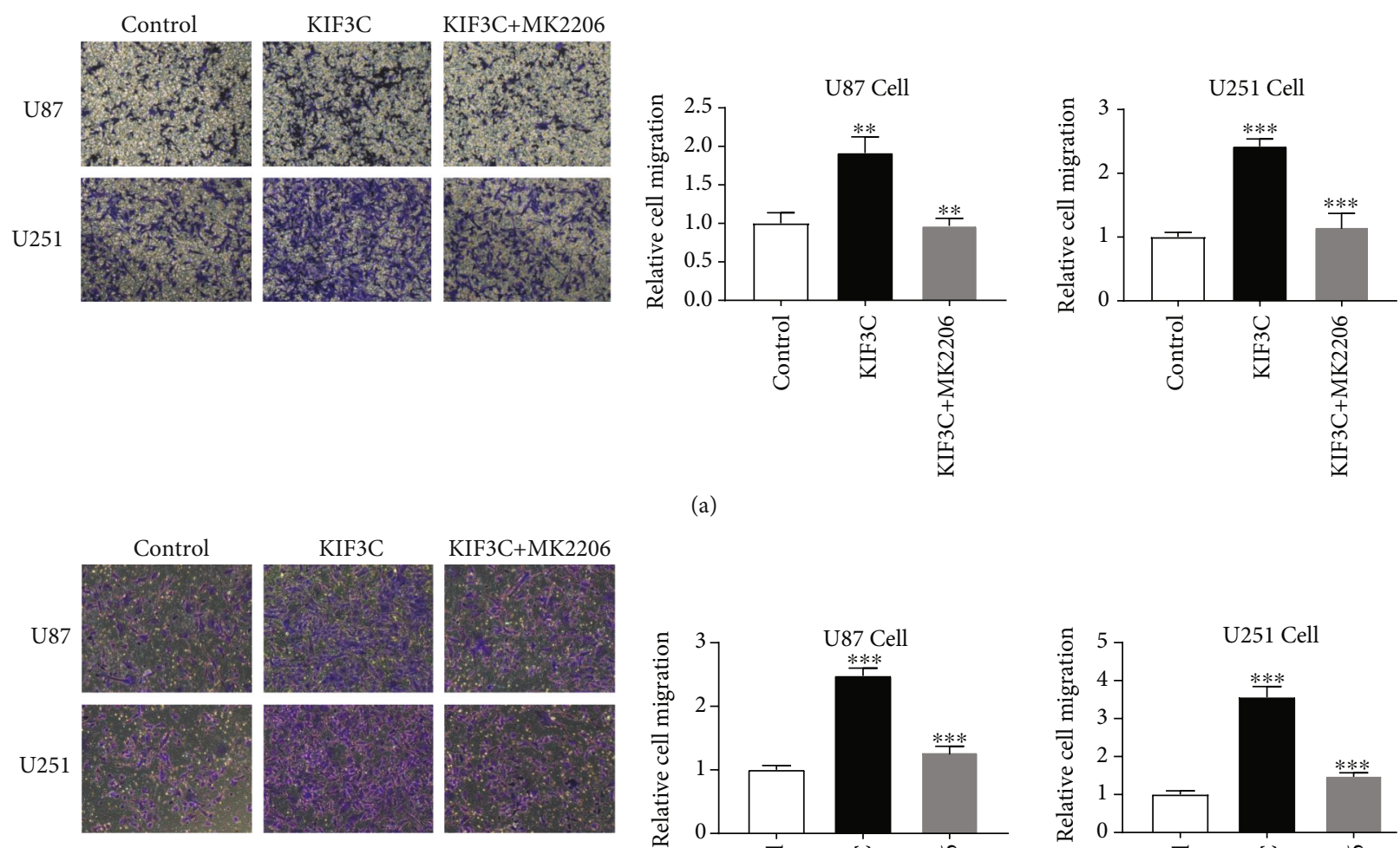

(a)
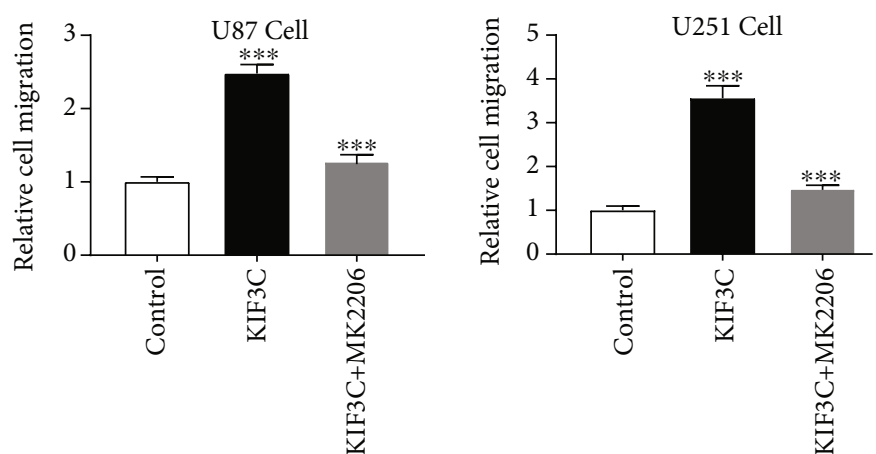

(b)
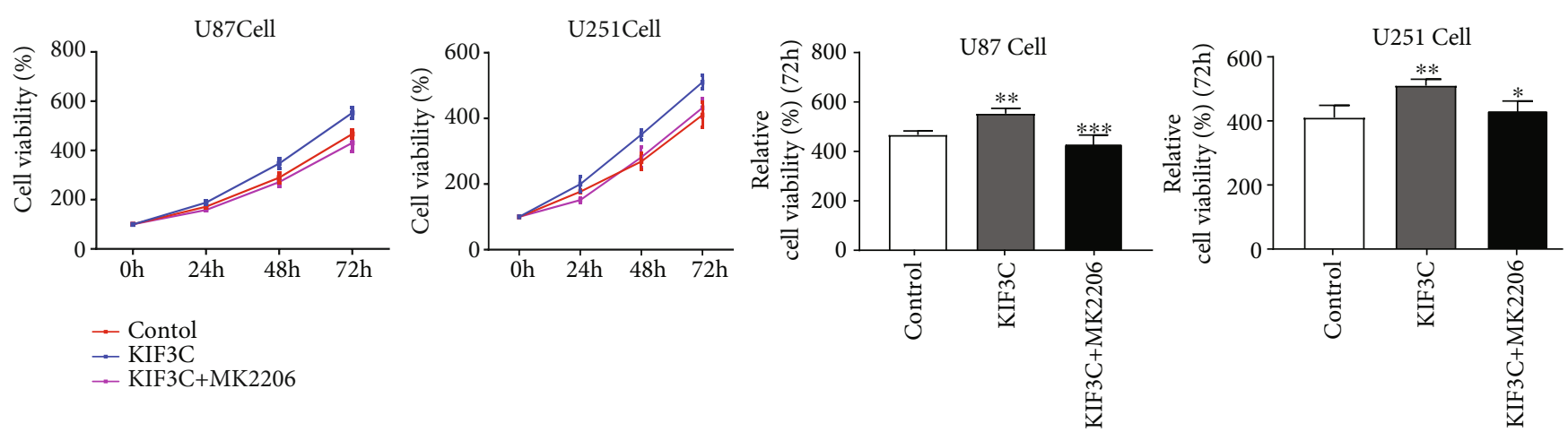

(c)

Figure 5: Continued. 

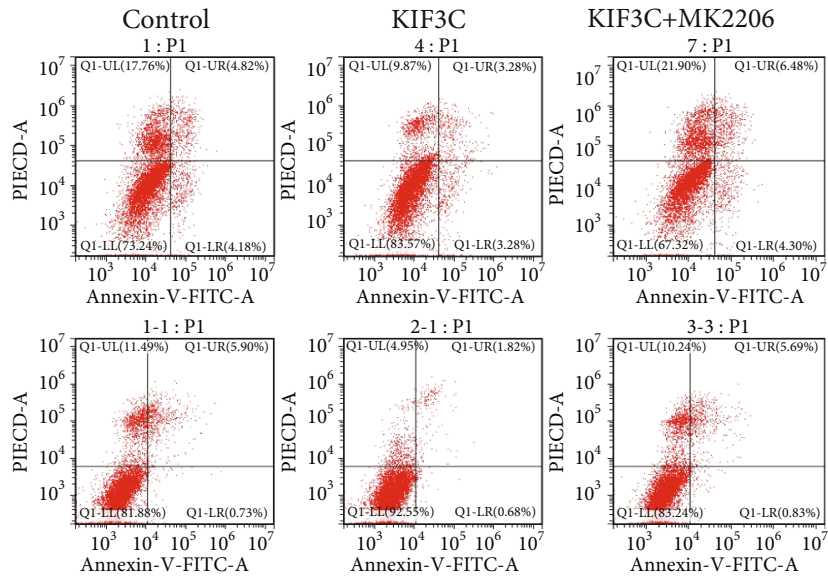
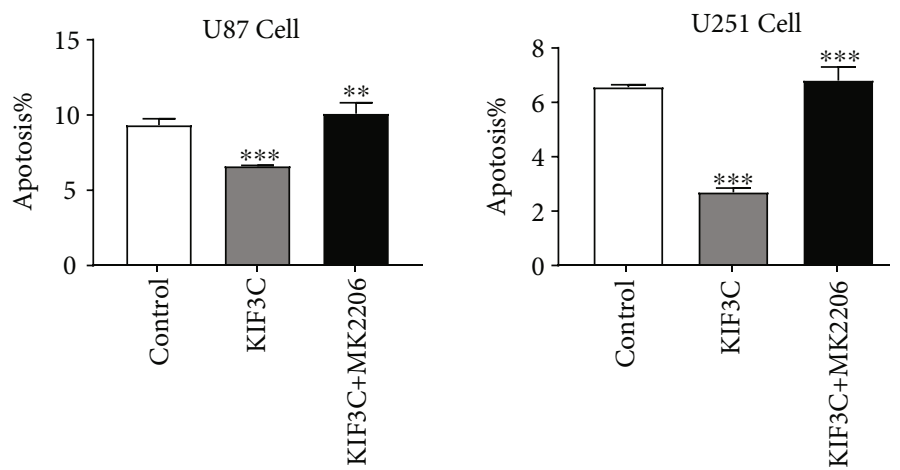

(d)

FIGURE 5: AKT inhibitor inhibits migration, invasion, and proliferation and enhances apoptosis of KIF3C-overexpressing cell lines. (a) Representative pictures of cell migration (left panel, magnification: 100x) and quantification of cell migration (right panel) (b) invasion, (c) proliferation, and (d) apoptosis in vitro. Data are expressed as the mean $\pm \mathrm{SD}$. Each experiment was repeated three times. ${ }^{*} P<0.05$, ${ }^{* *}$ $P<0.01$, and ${ }^{* * *} P<0.001$.

the PI3K/AKT pathway was affected by KIF3C in U87 and U251 cells. Western blotting results showed that in the glioma cell lines, the KIF3C downregulation group exhibited reduced levels of PI3K and phosphorylated AKT (Ser473). In addition, the KIF3C overexpression group had elevated levels of PI3K and phosphorylated AKT (Ser473), while the expression of total AKT protein did not change significantly (Figures 4(a) and 4(b)).

We then treated U87 and U251 cells with the AKT inhibitor MK-2206 (Selleck Chemicals, USA) to investigate whether AKT phosphorylation could mediate KIF3Cinduced glioma progression. We found that treatment of cells with MK-2206 reduced proliferation, migration, and invasion and promoted cell apoptosis (Figures 5(a)-5(d)). In addition, treatment of glioma cells with an AKT inhibitor suppressed the expression levels of $\mathrm{N}$-cadherin and p-AKT in KIF3C-overexpressing cells (Figures 6(a) and 6(b)). Altogether, our data suggest that KIF3C is probably involved in the PI3K/AKT pathway and can induce EMT in glioma cell lines.

\section{Discussion}

Various studies have demonstrated the significant role of KIFs in cell division, intracellular transport, and cellular morphogenesis [5-8]. However, most of these studies focused on the structure and function of KIFs in normal cells. In recent years, several studies have identified the role of KIFs (KIF3A and KIF3B) in human cancers, confirming the effect of kinesin on the proliferation or invasion of tumor cells [14, $16,21]$. Since KIF3C is expressed in the CNS, we investigate whether KIF3C has a potential role in glioma.

In this study, we conducted comprehensive research in vitro to explore the function of KIF3C in glioma. We first found that overexpression of KIF3C promoted cell proliferation, migration, and invasion and suppressed cell apoptosis, while silencing of KIF3C had the opposite effects on both gli- oma cell lines. Our results were consistent with those of a previous study in breast cancer cells, indicating the potential clinical value of KIF3C in glioma [21].

Previous evidence has suggested that KIF members influence the function of different human tumor cells through various signaling pathways. KIF3A promotes cell proliferation and invasion in advanced prostate cancer via the $W n t$ signaling pathway [14]. The downregulation of KIF3C expression inhibits tumor growth and metastasis in breast cancer by inhibiting TGF- $\beta$ signaling [21]. It has been reported that the PI3K/AKT pathway regulates tumor cell survival, growth, motility, angiogenesis, and metabolism in a variety of cancers, including GBM $[25,26]$. Inhibition of the PI3K/AKT pathway may result in GBM cell death and slow tumor progression $[27,28]$. It is reported that KIF3C was related to the PI3K/AKT pathway, which lacked a confirmatory experiment [29]. Therefore, we investigated whether KIF3C was involved in the PI3K/AKT pathway in glioma cells. In this study, western blotting results showed that overexpression of KIF3C elevated the levels of PI3K and p-AKT, while silencing of KIF3C reduced the levels of PI3K and pAKT. In addition, EMT is identified as a driver of invasion and metastasis in different types of epithelial cancers [30]. Our data supported that overexpression of KIF3C could increase the expression of $\mathrm{N}$-cadherin, vimentin, snail, and slug to promote EMT in vitro, while silencing of KIF3C had the opposite effects. Furthermore, we found that treatment of U87 and U251 cells with the AKT inhibitor MK-2206 reduced proliferation, migration, and invasion and promoted cell apoptosis in vitro. Treatment of glioma cells with an AKT inhibitor suppressed the expression levels of $\mathrm{N}$ cadherin and p-AKT in KIF3C-overexpressing cells. Taken together, these results indicate that KIF3C may be involved in the PI3K/AKT pathway and induce EMT in glioma cells.

Studying the distribution of KIF3C may provide important clues as to the possible functions of this molecule. KIF3C is overexpressed in breast cancer tissues, and such high 


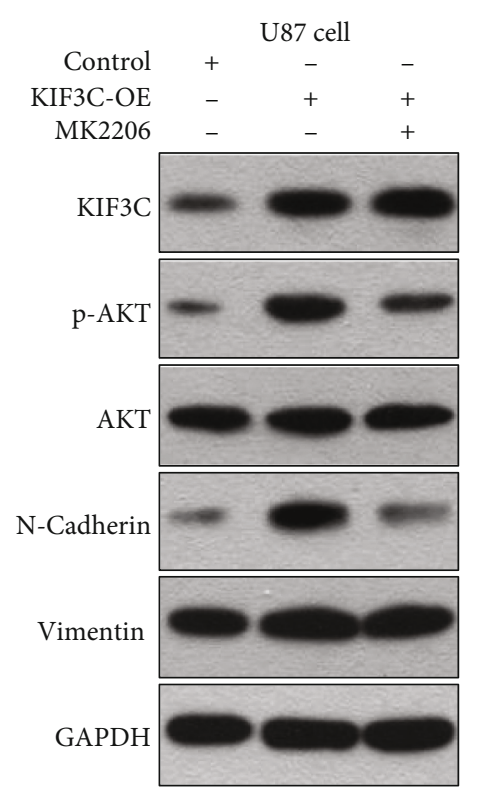

(a)

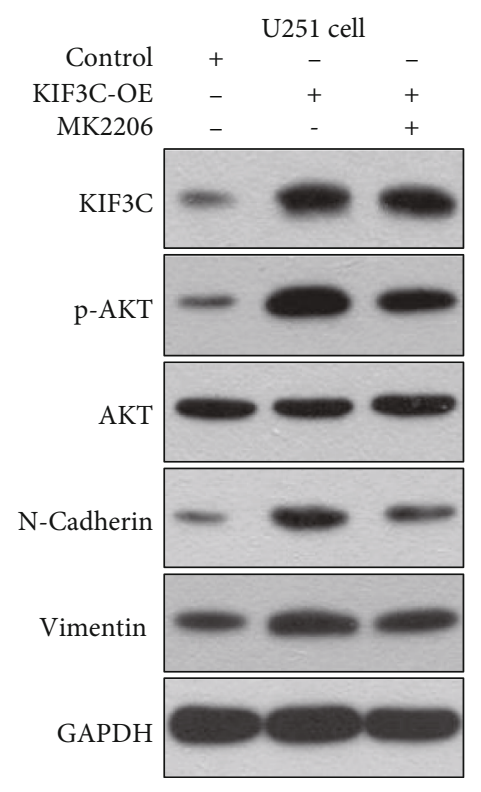

(b)

Figure 6: The AKT inhibitor MK2206 suppressed EMT in KIF3C-overexpressing cells. (a, b) Western blotting showed that N-cadherin was inhibited by the AKT inhibitor MK2206 in both KIF3C-overexpressing U87 and U251 cell lines.

KIF3C expression is also associated with tumor recurrence and lymph node metastasis [21]. However, in the CNS, the KIF3C peptide can efficiently induce glioma-reactive cytotoxic $\mathrm{T}$ lymphocytes (CTLs) from patients, indicating an effective peptide-based immunotherapy for glioma patients [31]. Of course, the role of this protein should be further investigated before clinical use. The high expression of $\mathrm{KIF} 3 \mathrm{C}$ in the nervous system during ontogenesis and its upregulation during neuronal differentiation have been observed and identified [20]. As for the lack of relevant research, we hypothesize that the expression level of KIF3C in different-grade gliomas (grades I to IV) may vary because of differentiation and dedifferentiation. Further study will explore the expression and function of KIF3C in high- and low-grade glioma tissues and in vivo.

\section{Conclusion}

We first confirm that overexpression of KIF3C promotes proliferation, migration, and invasion and inhibits apoptosis in glioma cells. Regarding the mechanism by which KIF3C regulates these functions, our results provide clues that the PI3K/AKT pathway and EMT may be involved, which require further investigation. These findings suggest that KIF3C might serve as a potential biomarker for further basic research or clinical management of glioma.

\section{Data Availability}

The datasets generated and/or analyzed during the present study are available from the corresponding author upon reasonable request.

\section{Conflicts of Interest}

The authors declare that they have no conflicts of interest.

\section{Authors' Contributions}

CYQ and CX designed the experiments. ZCS analyzed the data. LLD performed the experiments. ZXY revised the manuscript. ZH prepared the figures. GY wrote the paper. All authors read and approved the final manuscript. Yang Gao, Hui Zheng, and Liangdong Li contributed equally to this work.

\section{Acknowledgments}

This study is supported by grants from the Foundation of Shanghai Anti-Cancer Association (No. SACA-CY19C05), the National Natural Science Foundation of China (No. 81802494), and the Foundation of Shanghai Municipal Health Bureau (No. 20204Y0262).

\section{References}

[1] D. A. Jaoude, J. A. Moore, M. B. Moore, P. Twumasi-Ankrah, E. Ablah, and D. F. Moore, "Glioblastoma and increased survival with longer chemotherapy duration," Kansas Journal of Medicine, vol. 12, no. 3, pp. 65-69, 2019.

[2] A. Olar and K. D. Aldape, "Using the molecular classification of glioblastoma to inform personalized treatment," The Journal of Pathology, vol. 232, no. 2, pp. 165-177, 2014.

[3] A. Azab, N. Alsayegh, O. Kashkari et al., "Factors affecting survival in glioblastoma: a 10-year single-center experience from Saudi Arabia," The Gulf Journal of Oncology, vol. 1, no. 33, pp. $58-63,2020$. 
[4] T. Saito, K. Sugiyama, Y. Takeshima et al., "Prognostic implications of the subcellular localization of survivin in glioblastomas treated with radiotherapy plus concomitant and adjuvant temozolomide," Journal of Neurosurgery, vol. 128, no. 3, pp. 679-684, 2018.

[5] L. S. Goldstein and Z. Yang, "Microtubule-based transport systems in neurons: the roles of kinesins and dyneins," Annual Review of Neuroscience, vol. 23, no. 1, pp. 39-71, 2000.

[6] J. T. Kevenaar, S. Bianchi, M. van Spronsen et al., "Kinesinbinding protein controls microtubule dynamics and cargo trafficking by regulating kinesin motor activity," Current Biology, vol. 26, no. 7, pp. 849-861, 2016.

[7] N. Hirokawa and Y. Noda, "Intracellular transport and kinesin superfamily proteins, KIFs: structure, function, and dynamics," Physiological Reviews, vol. 88, no. 3, pp. 1089-1118, 2008.

[8] N. Hirokawa, S. Niwa, and Y. Tanaka, "Molecular motors in neurons: transport mechanisms and roles in brain function, development, and disease," Neuron, vol. 68, no. 4, pp. 610$638,2010$.

[9] J. Malicki and J. C. Besharse, "Kinesin-2 family motors in the unusual photoreceptor cilium," Vision Research, vol. 75, pp. 33-36, 2012.

[10] Z. Yang, E. A. Roberts, and L. S. B. Goldstein, "Functional analysis of mouse kinesin motor Kif3C," Molecular and Cellular Biology, vol. 21, no. 16, pp. 5306-5311, 2001.

[11] J. Teng, T. Rai, Y. Tanaka et al., "The KIF3 motor transports $\mathrm{N}$-cadherin and organizes the developing neuroepithelium," Nature Cell Biology, vol. 7, no. 5, pp. 474-482, 2005.

[12] J. R. Marszalek, X. Liu, E. A. Roberts et al., "Genetic evidence for selective transport of opsin and arrestin by kinesin-II in mammalian photoreceptors," Cell, vol. 102, no. 2, pp. 175$187,2000$.

[13] L. Lamri, W. K. Twan, T. A. Katoh et al., "Ciliogenesis-coupled accumulation of IFT-B proteins in a novel cytoplasmic compartment," Genes to Cells, vol. 24, no. 11, pp. 731-745, 2019.

[14] Z. Liu, R. E. Rebowe, Z. Wang et al., "KIF3a promotes proliferation and invasion via Wnt signaling in advanced prostate cancer," Molecular Cancer Research, vol. 12, no. 4, pp. 491503, 2014.

[15] A. Becher, T. Eiseler, M. Porzner et al., "The armadillo protein p0071 controls KIF3 motor transport," Journal of Cell Science, vol. 130, no. 19, pp. 3374-3387, 2017.

[16] X. Huang, F. Liu, C. Zhu et al., "Suppression of KIF3B expression inhibits human hepatocellular carcinoma proliferation," Digestive Diseases and Sciences, vol. 59, no. 4, pp. 795-806, 2014.

[17] V. Muresan, T. Abramson, A. Lyass et al., "KIF3C and KIF3A form a novel neuronal heteromeric kinesin that associates with membrane vesicles," Molecular Biology of the Cell, vol. 9, no. 3, pp. 637-652, 1998.

[18] M. Sardella, F. Navone, M. Rocchi et al., "KIF3C, a novel member of the kinesin superfamily: sequence, expression, and mapping to human chromosome 2 at 2p23," Genomics, vol. 47, no. 3, pp. 405-408, 1998.

[19] Z. Yang and L. S. Goldstein, "Characterization of the KIF3C neural kinesin-like motor from mouse," Molecular Biology of the Cell, vol. 9, no. 2, pp. 249-261, 1998.

[20] L. F. Gumy, D. J. Chew, E. Tortosa et al., “The kinesin-2 family member KIF3C regulates microtubule dynamics and is required for axon growth and regeneration," The Journal of Neuroscience, vol. 33, no. 28, pp. 11329-11345, 2013.
[21] C. Wang, C. Wang, Z. Wei et al., "Suppression of motor protein KIF3C expression inhibits tumor growth and metastasis in breast cancer by inhibiting TGF- $\beta$ signaling," Cancer Letters, vol. 368, no. 1, pp. 105-114, 2015.

[22] F. Navone, G. G. Consalez, M. Sardella et al., "Expression of KIF3C kinesin during neural development and in vitro neuronal differentiation," Journal of Neurochemistry, vol. 77, no. 3, pp. 741-753, 2001

[23] X. Li, C. Wu, N. Chen et al., "PI3K/Akt/mTOR signaling pathway and targeted therapy for glioblastoma," Oncotarget, vol. 7, no. 22 , pp. 33440-33450, 2016.

[24] H. F. Zhao, J. Wang, W. Shao et al., "Recent advances in the use of PI3K inhibitors for glioblastoma multiforme: current preclinical and clinical development," Molecular Cancer, vol. 16, no. 1, pp. 100-116, 2017.

[25] A. Sami and M. Karsy, "Targeting the PI3K/AKT/mTOR signaling pathway in glioblastoma: novel therapeutic agents and advances in understanding," Tumor Biology, vol. 34, no. 4, pp. 1991-2002, 2013.

[26] K. D. Courtney, R. B. Corcoran, and J. A. Engelman, "The PI3K pathway as drug target in human cancer," Journal of Clinical Oncology, vol. 28, no. 6, pp. 1075-1083, 2010.

[27] B. Wojtas, B. Gielniewski, K. Wojnicki et al., "Gliosarcoma is driven by alterations in PI3K/Akt, RAS/MAPK pathways and characterized by collagen gene expression signature," Cancers, vol. 11, no. 3, p. 284, 2019.

[28] H. A. Burris, "Overcoming acquired resistance to anticancer therapy: focus on the PI3K/AKT/mTOR pathway," Cancer Chemotherapy and Pharmacology, vol. 71, no. 4, pp. 829842, 2013.

[29] Y. Gao, L. Li, H. Zheng et al., "KIF3C is associated with favorable prognosis in glioma patients and may be regulated by PI3K/AKT/mTOR pathway," Journal of Neuro-Oncology, vol. 146 , no. 3, pp. 513-521, 2020.

[30] D. J. Burgess, "circulating and dynamic EMT," Nature Reviews Cancer, vol. 13, no. 3, p. 149, 2013.

[31] M. Harada, Y. Ishihara, K. Itoh, and R. Yamanaka, "Kinesin superfamily protein-derived peptides with the ability to induce glioma-reactive cytotoxic T lymphocytes in human leukocyte antigen-A24+ glioma patients," Oncology Reports, vol. 17, no. 3, pp. 629-636, 2007. 